\title{
Molecular Mechanism of EGFR Signaling Pathway Mediating Proliferation and Migration of U251 Glioma Cell Line
}

\section{Linlin YUE1 ${ }^{1}$, Ling LI¹, Bin WANG², Xiangmin YU1', Lingling DING1, Linlin Wang ${ }^{1}$, Yingying Li ${ }^{1}$}

\author{
${ }^{1}$ Department of Histology and Embryology, Qingdao University Medical College Qingdao 266071, China \\ ${ }^{2}$ Department of Pathogeny Biology, Qingdao University Medical College, Qingdao 266071, China
}

\begin{abstract}
The EGFR, epidermal growth factor recepor, is a $170 \mathrm{kDa}$ transmembrane glycoprotein and belongs to receptor tyrosine kinases family. Actual evidence shows that overexpression of EGFR is involved in $50 \%-70 \%$ of various diseases. However, there is limited evidence regarding the role of EGFR in the tumorigenesis of human gliomas. In the present study, its effects on U251 cell proliferation and migration were examined using CCK and scratch-wound assay. We found that AG1478 inhibit EGF-induced U251 cells proliferation and migration. Moreover, it was demonstrated that the downregulation of p-EGFR in U251 glioma cells inhibited the expression of P-Akt. Taken together, these findings suggest that the EGFR/Akt signaling pathway involved in the regulation of U251 glioma cells proliferation and migration. It's mechanism is possible that after the EGFR/Akt signaling pathway activated, caused the high expression of downstream proteins.
\end{abstract}

Keyword: glioma; epidermal growth factor; proliferation; migration

\section{Introduction}

Malignant gliomas are the most common and deadly primary brain tumors of the central nervous system[1]. Glioblastoma multiforme (GBM) is the most malignant and common type of glioma, which arises from astrocytes with poor differentiation and is associated with a median survival of approximately 10-14 months $[2,3]$. Rapid proliferation and diffuse brain metastasis are the histopathological hallmarks of these tumors and are likely to determine the unfavorable prognosis[4]. Therefore, the recurrence of gliomas remains inevitable. Novel therapeutic targets, based on the identification of molecular events key to carcinogenesis and tumor progression, are necessary in order to improve the overall outcome of patients with GBM[5].

The EGFR, also named c-erb-B1, is a $170 \mathrm{kDa}$ transmembrane glycoprotein and belongs to receptor tyrosine kinases family[6]. Existing evidence suggests that increased expression of EGFR is involved in 50\% $70 \%$ of various diseases[6-8]. EGFR participates in many aspects of cell biology and can be activated not only by many protein kinases, such as integrins, cytokine receptors, and adhesion receptors but also by ligand-induced signaling. Although overexpression of 
EGFR has been found in various diseases[9-13], the precise role that EGFR plays in the proliferation and migration of glioma have not been extensively studied. In this study, we examined how EGFR affects the proliferation and migration of glioma cells by changing EGFR pathway status in the glioma cells (U251 cells).

\section{Materials and methods}

Reagents and antibodies

Epidermal growth factor (EGF) and Tyrphostin AG1478 were purchased from sjgma (USA).Primary antibodies for Phospho-Akt(Ser473)(\#4060), Akt (\#4685), p-EGFR (Tyr1173) (\#4407), EGFR (\#4267) and anti-rabbit IgG horseradish peroxidase-conjugated secondary antibodies were purchased from Cell Signaling Technology (Beverly, MA, USA). Cell counting kit (CCK) was purchased from Dojindo Laboratories (Kumamoto, Japan). Dulbecco's modified Eagle's medium (DMEM) and Fetal bovine serum (FBS) were obtained from GIBCO (Life Technologies, USA). Trypsin-EDTA was purchased from Beyotime Biotechnology (shanghai, China). PVDF membranes were obtained from Millipore (Billerica, MA, USA). BCA Protein assay kit (\#PC0020), RIPA Lysis Buffer (\#R0020), PMSF(\#P0100), sodium dodecyl sulfatepolyacrylamide gel electrophoresis (SDS-PAGE,\#P1015) and Protein phosphatase inhibitor(All-in-one,100×)(\#P1260) were purchased from Beijing Solarbio Science \&Technology (Beijing, China).

\section{Cell liens and cell culture}

The glioma cell lines U251 that were used in this study were obtained from the Institute of Biochemistry and Cell Biology (Shanghai Institutes for Biological Sciences, Chinese Academy of Science, Shanghai, China). The cells were cultured in Dulbecco's modified Eagle's medium (DMEM) supplemented with $10 \%$ FBS and maintained in a humidified atmosphere of 5\% $\mathrm{CO}_{2}$ at $37^{\circ} \mathrm{C}$. The medium was changed every other day. Cells were passed when $80 \%$ of the bottle wall was covered.

\section{Scratch-wound assay}

Cell migration was examined using scratch-wound assays. Cells were seeded into 6-well plates at $90 \%$ confluency. Monolayers were wounded with a $200 \mu \mathrm{l}$ sterile pipette tip and rinsed with phosphate-buffered saline (PBS) 3 times to remove any floating cells. Then the cells were incubated in the absence or presence of EGF $(100 \mathrm{ng} / \mathrm{mL})$ with or without AG1478 (10 $\mu \mathrm{M}$, was added $1 \mathrm{~h}$ before the EGF) for $24 \mathrm{~h}$. The wound location in the 6-well plates was marked. Images were captured at 0 and $24 \mathrm{~h}$ after wound application. The level of cell migration into the wound was quantified as the migration rate compared against the cells at $0 \mathrm{~h}$ for each group.

\section{Proliferation assays}

The proliferation rates of U251 treated absence or presence of EGF (100 ng/mL) with or without AG1478 (10 $\mu \mathrm{M}$, was added $1 \mathrm{~h}$ before the EGF) were measured by cell counting kit (CCK) assay in accordance with the manufacturer's instructions. A total of $5 \times 10^{3} \mathrm{U} 251$ cells growing on a 96-well plate were treated with anticipated concentration of EGF and AG1478 for 12, 24,36 and $48 \mathrm{~h}$ in triplicate. Control group was treated with medium. $10 \mu \mathrm{L}$ of CCK solution was added to each well at the end of incubation. The optical density (OD) values were measured by an enzyme-labeled instrument at $450 \mathrm{~nm}$. Relative cell viability was calculated using the following formula: relative cell viability $(\%)=[(A s-A b) /(A c-A b)]$, where $A s, A b$, and Ac denoted the absorbance of experimental group, blank group, and control group, respectively.

\section{Western blot analysis}

Total cell lysates from different experiments were obtained by lysing the cells in RIPA buffer supplemented with a cocktail of protease inhibitors and protein phosphatase inhibitor. The protein concentration was determined using a BCA Protein assay kit following the manufacturer's protocol. Equal amounts of soluble proteins were separated by $10 \%$ SDS-PAGE and transferred to PVDF membranes. The nonspecific protein binding sites on the membranes 
were then blocked with $5 \%$ dried nonfat milk in Tris-buffered saline $(20 \mathrm{mM}$ Tris and $0.5 \mathrm{M} \mathrm{NaCl}, \mathrm{pH}$ 7.6) with $0.1 \%$ Tween-20 (TBST) at room temperature for $2 \mathrm{~h}$. Membranes were blocked and incubated with primary antibodies overnight at $4{ }^{\circ} \mathrm{C}$ before washed and incubated with horseradish conjugated secondary antibody for $1 \mathrm{~h}$ at room temperature. The signal intensity was determined using gel analysis software, ImageJ.

\section{Statistical analysis}

All data are presented as the means \pm SD. The experiments were repeated three times. All statistical analyses were performed using a two-tailed Student's t-test in SPSS 12.0 software. $P<0.05$ was considered to indicate a statistically significant difference.

\section{Result}

1. AG1478 inhibit EGF-induced U251 cells migration

We performed the scratch-wound assay in order to explore the effect of EGF upregulation and downregulation on glioma cell migration in vitro. After treatment with hEGF, U251 cells were found to have significantly enhanced migration into the wound, while cells treated with AG1478 and EGF had less migratory ability. These results demonstrated that AG1478 suppressed EGF-induced U251 cell migration. Fig1

A

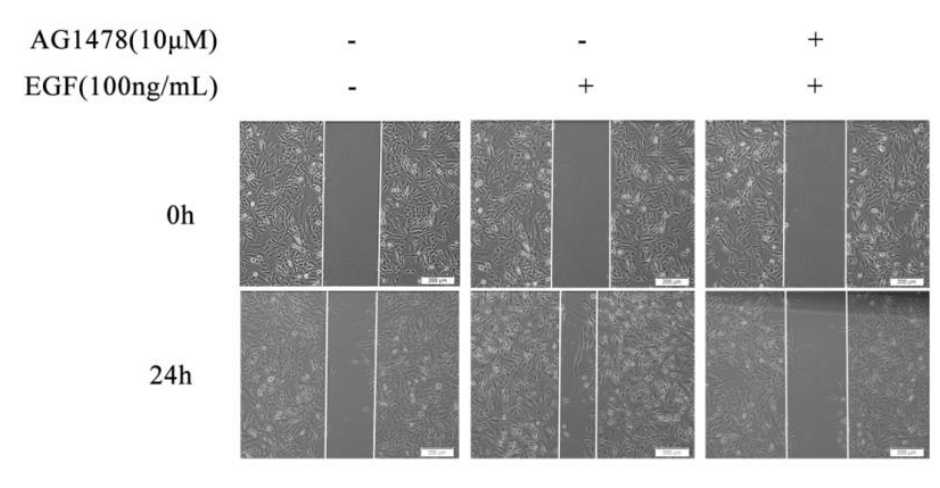

B

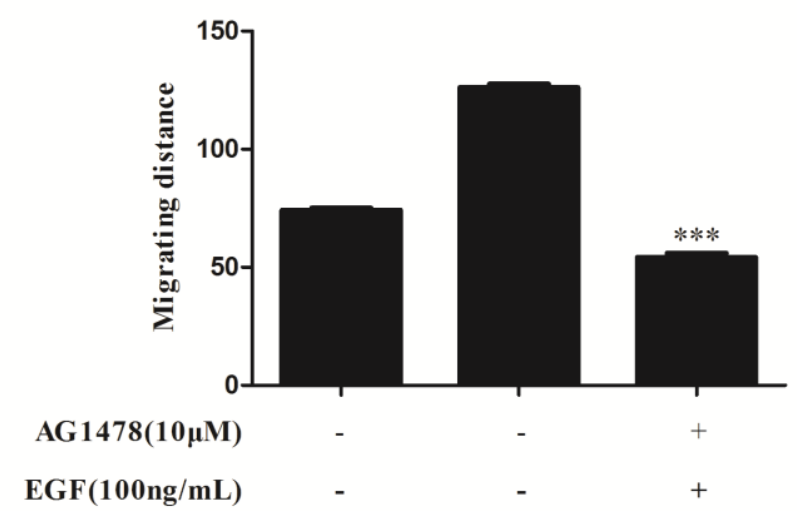

Effects of AG1478 on the EGF-induced migration of U251 glioma cells cells. (A) Confluent monolayers were scratched and incubated in the absence or presence of EGF $(100 \mathrm{ng} / \mathrm{mL})$ with or without AG1478 $(10 \mu \mathrm{M})$ for $24 \mathrm{~h}$. The images were captured at
0 and $24 \mathrm{~h}$ after wounding (magnification, $\mathrm{x} 200$ ). These figures are representative of 3 separate experiments. (B) The inhibition of EGF-stimulated migration by AG1478. Values are mean \pm SD. $* * * P<0.001$ compared with the EGF-treated group. 


\section{AG1478 inhibit EGF-induced U251 cells}

\section{Proliferation}

We performed the cell proliferation assay in order to explore the effect of EGF upregulation and downregulation on glioma cell proliferation in vitro. The cell proliferation rate was clearly increased in the
EGF treatment group compared with the NC groups. By contrast, the cell proliferation rate was inhibited following treatment with the EGFR inhibitor. This finding indicates that AG1478 significantly inhibits EGF-stimulated proliferation of glioma cells.

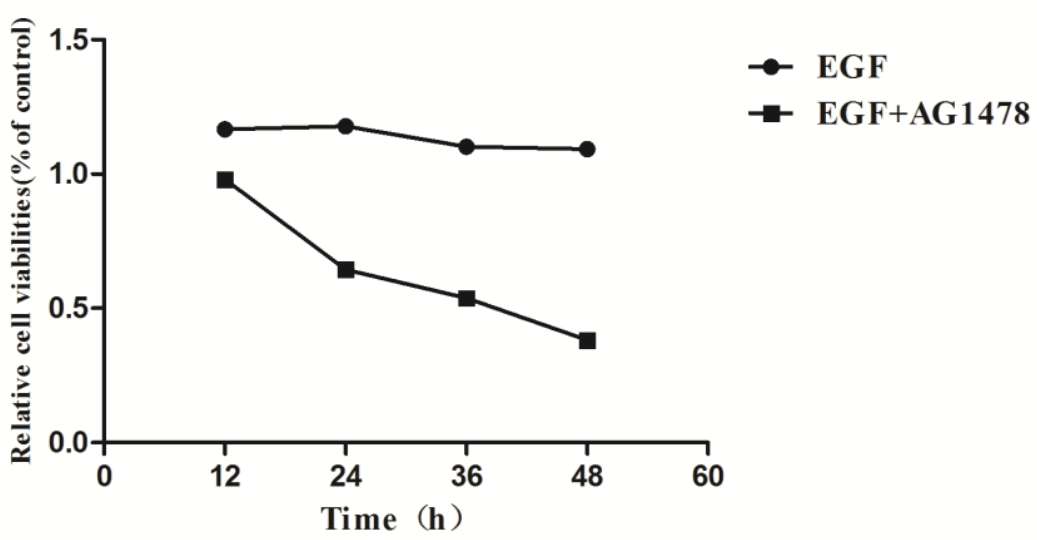

Effects of AG1478 on the EGF-induced proliferation of U251glioma cells. Cells were treated with EGF with or without AG1478 for 12, 24, 26 and $48 \mathrm{~h}$. The result is represented by the relative cell viability.

\section{AG1478 prevents EGF-induced EGFR/Akt} signal transduction

Here, we aimed to determine whether or not AG1478 has inhibitory effects on p-EGFR and P-akt expression or its signal transduction. We observed that the expression of EGFR and akt remained unchanged after exposure to EGF with or without AG1478 in U251 cells. Previous studies have indicated that P-EGFR activation triggers EGFR transactivation in U251cells, thus we sought to evaluate the influence of AG1478 in EGFR phosphorylation upon EGF stimulation. When cells were treated with AG1478 in the presence of EGF, there was a significant decrease in EGFR phosphorylation (Tyr1173). AG1478 triggers EGFR phosphorylation leading to the phosphorylation of Akt and activation of the PI3K/Akt transduction pathway. Consequently, the EGFR/Akt signaling pathway involved in the regulation of U251 glioma cells proliferation. Its mechanism is possible that after the EGFR/Akt signaling pathway activated, caused the high expression of downstream proteins.

A

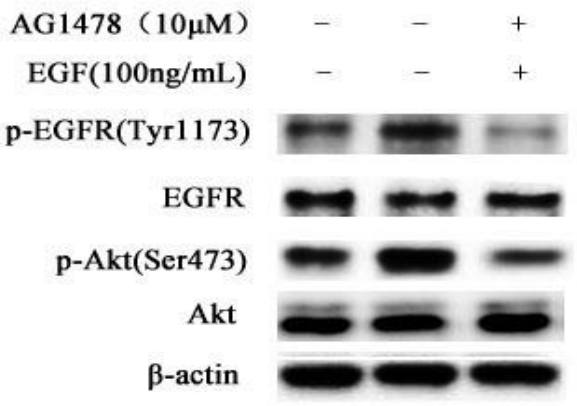


B

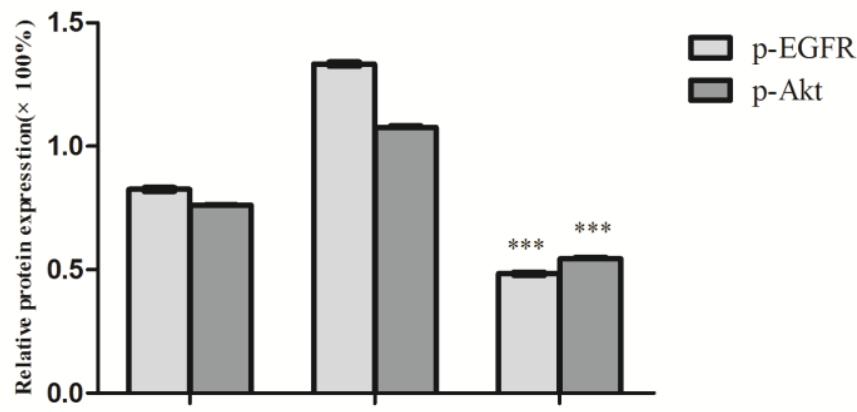

\section{AG1478(10 $\mu M)$ \\ $\operatorname{EGF}(100 \mathrm{ng} / \mathrm{mL})$}

Effect of AG1478 on EGF-stimulated activation of the EGFR /Akt signaling pathway. The cells were harvested after EGF (100 ng/mL) with or without AG1478 $(10 \mu \mathrm{M})$ treatment for $24 \mathrm{~h}$. (A) Western blot assay was used to examine the expression of p-EGFR and P-Akt. $\beta$-actin was used as a loading control. (B) The totaland phosphorylated protein levels of EGFR and Akt. $* * * P<0.0001$, significantly different from the EGF-treated group.

\section{Conclusion}

Gliomas are the most common primary tumors of the central nervous system with glioblastomas as the most malignant entity[1]. Despite progress in glioma therapy regimens, such as surgery, radiation, chemotherapy or combined modalities, the prognosis for malignant glioma patients remains dismal[14, 15], and most patients with glioma die within two years of diagnosis[16]. Therefore, extensive study of the biological characteristics of gliomas is necessary in order to identify an effective treatment capable of suppressing the proliferation of glioma cells. In the present study, AG1478 treatment inhibited cell migration in response to EGF stimulation. Furthermore, it significantly decreased EGF-promoted proliferation ability. These results imply that AG1478 may possess EGFR signaling pathway to suppress EGF-induced migration and proliferation of U251 cells in vitro.

EGFR is a type of receptor tyrosine kinases (RTKs), playing a central role in cell division, migration, adhesion, differentiation and apoptosis[17, 18]. GFR alteration, including overexpression or gene amplification, is the most frequent form of genetic mutation, occurred in 40-50\% of glioblastomas[19, 20]. EGFR comprises of extracellular ligand binding domain, transmembrane domain and intracellular tyrosine kinase domain. Upon binding to various of ligands, such as EGF, EGFR is activated through homodimerization or heterdimerization on the cell surface and subsequently leads to the phosphorylation of its intracellular tyrosine kinase domain[21]. The activation of EGFR results in activation of multiple downstream signal transduction pathways such as PI3K/Akt/mTOR pathway[22]. In the past study, EGF can promote prostate cancer growth and proliferation by activating EGFR-PI3K / AKT signaling pathway, but the function of it was inhibited while blocking the EGFR gene. Domestic scholars have reported that activation of EGFR signaling pathway can enhance overexpression of MMP-2 and MMP-9 and promote invasion of colon cancer cells, which effect can be EGFR Inhibitors block[23]. In this study, We observed that the expression of EGFR and akt remained unchanged after exposure to EGF with or without AG1478 in U251 cells. When cells were treated with AG1478 in the presence of EGF, there was a significant decrease in EGFR phosphorylation (Tyr1173). AG1478 triggers EGFR phosphorylation leading to the phosphorylation of Akt and activation of the PI3K/Akt 
transduction pathway.

Taken together, These findings suggest that the EGFR/PI3K/Akt signaling pathway plays a key role in the development of glioma. Consequently, the EGFR/PI3K/Akt signaling pathway involved in the regulation of $\mathrm{U} 251$ glioma cells proliferation. Its mechanism is possible that after the EGFR/Akt signaling pathway activated, caused the high expression of downstream proteins.

\section{References}

1. Van Meir, E.G., et al., Exciting new advances in neuro-oncology: the avenue to a cure for malignant glioma. CA Cancer J Clin, 2010. 60(3): p. 166-93.

2. Louis, D.N., et al., The 2007 WHO classification of tumours of the central nervous system. Acta Neuropathol, 2007. 114(2): p. 97-109.

3. Wiedemeyer, R., et al., Feedback circuit among INK4 tumor suppressors constrains human glioblastoma development. Cancer Cell, 2008. 13(4): p. 355-64

4. Coras, R., et al., The peroxisome proliferator-activated receptor-gamma agonist troglitazone inhibits transforming growth factor-beta-mediated glioma cell migration and brain invasion. Mol Cancer Ther, 2007. 6(6): p. 1745-54.

5. $\mathrm{Wu}, \mathrm{Z}$., et al., Upregulation of miR-153 promotes cell proliferation via downregulation of the PTEN tumor suppressor gene in human prostate cancer. Prostate, 2013. 73(6): p. 596-604

6. Li, J.C., et al., Clinical significance of the expression of EGFR signaling pathway-related proteins in esophageal squamous cell carcinoma. Tumour Biol, 2014. 35(1): p. 651-7.

7. Jia, J., et al., The relation of EGFR expression by immunohistochemical staining and clinical response of combination treatment of nimotuzumab and chemotherapy in esophageal squamous cell carcinoma. Clin Transl Oncol, 2016. 18(6): p. 592-8.

8. $\mathrm{Mu}, \mathrm{L}$., et al., beta-Elemene enhances the efficacy of gefitinib on glioblastoma multiforme cells through the inhibition of the EGFR signaling pathway. Int J Oncol, 2016. 49(4): p. 1427-36.

9. Ohashi, S., et al., Recent Advances From Basic and Clinical Studies of Esophageal Squamous Cell Carcinoma. Gastroenterology, 2015. 149(7): p. 1700-15.

10. Bonaccorsi, L., et al., Gefitinib ('IRESSA', ZD1839) inhibits
EGF-induced invasion in prostate cancer cells by suppressing PI3 K/AKT activation. J Cancer Res Clin Oncol, 2004. 130(10): p. 604-14.

11. P, O.c., et al., The role of c-erbB receptors and ligands in head and neck squamous cell carcinoma. Oral Oncol, 2002. 38(7): p. $627-40$

12. Ellerbroek, S.M., et al., Phosphatidylinositol 3-kinase activity in epidermal growth factor-stimulated matrix metalloproteinase-9 production and cell surface association. Cancer Res, 2001. 61(5): p. 1855-61.

13. Yufen, X., et al., The role of EGFR-TKI for leptomeningeal metastases from non-small cell lung cancer. Springerplus, 2016. 5(1): p. $016-2873$

14. Normanno, N., et al., Epidermal growth factor receptor (EGFR) signaling in cancer. Gene, 2006. 366(1): p. 2-16.

15. Walbert, T. and K. Chasteen, Palliative and supportive care for glioma patients. Cancer Treat Res, 2015. 163: p. 171-84.

16. Siegel, R.L., K.D. Miller, and A. Jemal, Cancer statistics, 2015. CA Cancer J Clin, 2015. 65(1): p. 5-29.

17. Mellinghoff, I.K., et al., Molecular determinants of the response of glioblastomas to EGFR kinase inhibitors. N Engl J Med, 2005. 353(19): p. 2012-24.

18. Chakravarti, A., A. Dicker, and M. Mehta, The contribution of epidermal growth factor receptor (EGFR) signaling pathway to radioresistance in human gliomas: a review of preclinical and correlative clinical data. Int J Radiat Oncol Biol Phys, 2004. 58(3): p. 927-31.

19. Nishikawa, R., et al., A mutant epidermal growth factor receptor common in human glioma confers enhanced tumorigenicity. Proc Natl Acad Sci U S A, 1994. 91(16): p. 7727-31.

20. Szerlip, N.J., et al., Intratumoral heterogeneity of receptor tyrosine kinases EGFR and PDGFRA amplification in glioblastoma defines subpopulations with distinct growth factor response. Proc Natl Acad Sci U S A, 2012. 109(8): p. 3041-6.

21. Zhang, H., et al., ErbB receptors: from oncogenes to targeted cancer therapies. J Clin Invest, 2007. 117(8): p. 2051-8.

22. Huang, P.H., A.M. Xu, and F.M. White, Oncogenic EGFR signaling networks in glioma. Sci Signal, 2009. 2(87).

23. Li, X., et al., PI3K/Akt/mTOR signaling pathway and targeted therapy for glioblastoma. Oncotarget, 2016. 7(22): p. 33440-50. 